\title{
THE 2D ORIENTATION IS UNIQUE THROUGH PRINCIPAL MOMENTS ANALYSIS
}

\author{
João F. P. Crespo, Pedro M. Q. Aguiar \\ Institute for Systems and Robotics / IST, Lisboa, Portugal \\ aguiardisr.ist.utl.pt
}

\begin{abstract}
When comparing 2D shapes, a key issue is their normalization. Translation and scale are easily taken care of by removing the mean and normalizing the energy. However, defining and computing the orientation of a $2 \mathrm{D}$ shape is not so simple. In fact, although for elongated shapes the principal axis can be used to define one of two possible orientations, there is not such a tool for general shapes. As we show in the paper, previous approaches fail to compute the orientation of even noiseless observations of simple shapes. We address this problem. In the paper, we show how to uniquely define the orientation of an arbitrary 2D shape, in terms of what we call its principal moments. We further propose a new method to efficiently compute the shape orientation: Principal Moments Analysis. Besides the theoretical proof of correctness, we describe experiments demonstrating robustness to noise and illustrating with real images.
\end{abstract}

Index Terms - Image shape analysis, Moment methods

\section{INTRODUCTION}

Having to deal with unknown orientation in shape-based image analysis has proved to be very challenging. Consider two instances of a 2D shape described by an arbitrary set of 2D points obtained, e.g., from edges or corners, of differently oriented images. The challenge when comparing these two sets is that they are related by an unknown transformation that includes, simultaneously, a 2D rotation, due to different orientations, and a permutation, due to the absence of labels for the points. Iterative methods have been used to compute, in alternate steps, rotation and permutation: the Iterative Closest Point (ICP) algorithm [1], or its probabilistic versions based on Expectation-Maximization (EM), see, e.g., [2]. However, due to the non-convex nature of the problem, these approaches suffer from sensitivity to the initialization, exhibiting uncertain convergence.

Recently, researchers have proposed permutation-invariant representations for point sets. For example, [3] factors out unknown labels, by solving a convex optimization problem over the set of permutation matrices, and [4] proposes a permutation-invariant representation based on analytic functions. These approaches do not cope adequately with arbitrary orientations: [3] assumes the shape orientation is known and the representation in [4] is not invariant to rotation, requiring pairwise alignment. In this paper, we show how to obtain orientation-invariance in shape analysis, by rotating each shape of an angle that unambiguously defines its orientation.

The most straightforward method to define orientation uses the principal axis of the shape, obtained, e.g., through Principal Component Analysis (PCA). Although unable to provide a unique orientation, this method defines two possible orientations for elongated shapes. However, for shapes that do not have a well defined principal

Partially supported by FCT, under ISR/IST plurianual funding (POSC program, FEDER) and grant MODI-PTDC/EEA-ACR/72201/2006, and FET within the EU FP7, under the SIMBAD project (contract 213250). axis (rotationally symmetric shapes are an extreme example), PCAbased orientation is completely ambiguous. To deal with these ambiguities, researchers attempted to work with concepts like mirrorsymmetry axes [5], universal principal axis [6], and generalized principal axis [7]. In general, the motivation for these works is more on the definition of a "reasonable geometric orientation" than on the robust computation of a unique orientation angle for arbitrary shapes. Since rotationally symmetric shapes are particularly challenging, the detection of symmetry and fold number, by itself a relevant problem, has also received attention $[8,9]$.

The more theoretically sustained methods to compute orientation are based on the geometric moments of the points defining the shape. In particular, the so-called Complex Moments (CMs) were introduced in the eighties $[10,11]$. The elegance of these approaches comes from defining the orientation through the phase of a single $\mathrm{CM}$ of a particular order. In the late nineties, more general moments were proposed to deal with degenerate shapes [8], at a cost of dealing with several moments, chosen by tuning a free parameter index through search, and detecting rotational symmetry as an intermediate step. However, as we detail in Sec. 3, these methods do not cope with several shapes that lead to singular moments. In practice, this means the phase of these moments is sensitive to noise, leading to unstable estimates of orientation.

Other approaches require the exhaustive search for the angle maximizing a given orientation measure, e.g., [12], without any guarantee of uniqueness of the solution. Due to these difficulties, the search for 2D geometric invariance in image classification is still ongoing, see, e.g., $[4,13]$. In this paper, we present a new method to define and compute the unique orientation of any 2D shape. We use what we call the principal moments, which have the distinguishing feature of uniquely describing the $2 \mathrm{D}$ shape. We show that the phases of two of these moments unambiguously define the orientation of an arbitrary 2D shape (including rotationally symmetric ones). We propose an algorithm, Principal Moments Analysis (PMA), that computes 2D shape orientation by integrating the contributions of all (pairs of) moments. Besides theoretically sound, PMA results are robust to noise, as our experiments illustrate.

\section{PROBLEM DEFINITION}

Consider an arbitrary 2D shape described by $N$ points, thus by an $N$-dimensional complex vector $\mathbf{z}$, containing their coordinates:

$$
\mathbf{z}=\left[\begin{array}{llll}
z_{1} & z_{2} & \cdots & z_{N}
\end{array}\right]^{T} .
$$

It is straightforward to obtain invariance with respect to translation and scale, by removing the mean and normalizing the energy of $\mathbf{z}$.

A natural and the most common way to attempt to obtain orientation invariance consists in finding an angle $\theta(\mathbf{z})$ such that, through rotation, any shape $\mathbf{z}$ is brought to its "normalized" version

$$
\mathbf{w}(\mathbf{z})=\mathbf{z} e^{-j \theta(\mathbf{z})} \text {. }
$$


In fact, if the desired invariance is satisfied, i.e., if,

$$
\forall_{\phi}, \quad \mathbf{w}\left(\mathbf{z} e^{j \phi}\right)=\mathbf{w}(\mathbf{z}),
$$

the normalization in (2) produces a complete (a.k.a maximal) invariant, in the sense that two shape vectors lead to the same normalized version if and only if they contain (differently oriented versions of) the same shape. However, current methods to compute the shape orientation $\theta(\mathbf{z})$ either fail to process particular shapes (see examples in the following section) or do not guarantee (3).

The success of the normalization in (2) hinges then on finding an appropriate function $\theta: \mathbb{C}^{n} \rightarrow(-\pi, \pi]$ that unambiguously defines $\theta(\mathbf{z})$, the orientation of the shape $\mathbf{z}$. We now show that, to guarantee the desired invariance, it suffices that this function satisfies a natural condition: that the orientation of a rotated shape is the sum of the orientation of the original shape with the rotation angle. Formally,

$$
\forall_{\phi}, \quad \theta\left(\mathbf{z} e^{j \phi}\right)=\theta(\mathbf{z})+\phi .
$$

Simple manipulations show that (4) suffices to guarantee (3):

$$
\begin{aligned}
\mathbf{w}\left(\mathbf{z} e^{j \phi}\right) & =\mathbf{z} e^{j \phi} \exp \left(-j \theta\left(\mathbf{z} e^{j \phi}\right)\right) \\
& =\mathbf{z} e^{j \phi} e^{-j(\theta(\mathbf{z})+\phi)} \\
& =\mathbf{z} e^{-j \theta(\mathbf{z})}=\mathbf{w}(\mathbf{z})
\end{aligned}
$$

where (5) and (7) use the definition (2) and (6) uses (4).

\section{SHAPE MOMENTS}

To obtain an orientation function $\theta(\cdot)$ satisfying (4), we will use moments of the points describing the shape. Since image moments have been used in the past, we first overview momentbased estimation of orientation and motivate the need to revisit the problem. The usage of Complex Moments (CMs) to define orientation was proposed in [10]. CMs stand for compact representations of linear combination of ordinary (i.e., real) geometric moments. The CM of order $(p, q)$ of an image $g(x, y)$ is defined by $C_{p q}=\int_{-\infty}^{+\infty} \int_{-\infty}^{+\infty}(x+j y)^{p}(x-j y)^{q} g(x, y) d x d y$. In [10], the orientation is defined and computed by imposing the phase of one of the moments $C_{q+1, q}$ to be zero. When applying this method to a shape $\mathbf{z}$, i.e., to an image composed by $N$ mass points, the integral becomes a sum and the corresponding CMs are given by

$$
C_{q+1, q}(\mathbf{z})=\sum_{n=1}^{N}\left|z_{n}\right|^{2 q+1} e^{j \arg z_{n}} .
$$

Although the method just described is adequate to deal with shapes $\mathbf{z}$ that lead to a moment $C_{q+1, q}(\mathbf{z})$ with large magnitude, there are shapes for which this does not happen for any $q$. It was known that this is the case of rotationally symmetric shapes [10], but we now show it may also happen with general shapes. Consider $\mathbf{z}=[1, j,-j, \exp (j 2 \pi / 3), \exp (-j 2 \pi / 3)]^{T}$, as represented in the left plot of Fig. 1. For this shape, from (8), we obtain $C_{q+1, q}(\mathbf{z})=$ $e^{j 0}+e^{j \pi / 2}+e^{-j \pi / 2}+e^{j 2 \pi / 3}+e^{-j 2 \pi / 3}=0$, regardless of $q$. For $S$-fold rotationally symmetric shapes, [10] proposes to use the phase of one of the moments $C_{q+S, q}$. However, again, there are $S$-fold rotationally symmetric shapes for which these are all zero. For example, the 2-fold rotationally symmetric shape in the right plot of Fig. $1, \mathbf{z}=[ \pm 1, \pm \exp ( \pm j \pi / 3), \pm \exp ( \pm j \pi / 4)]^{T}$, leads to $C_{q+2, q}(\mathbf{z})=0, \forall_{q}$. Although these examples serve as mere illustrations of extreme cases, they also make clear that in practice it is
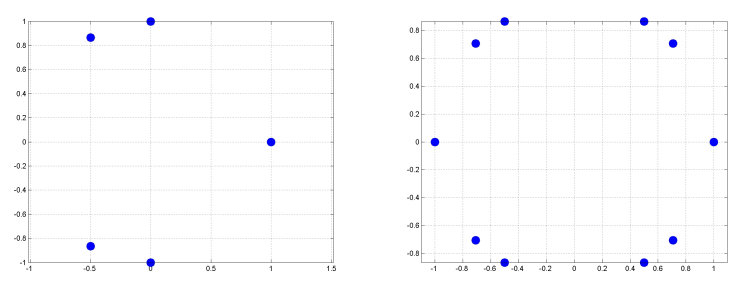

Fig. 1. Examples illustrating limitations of other methods (see text).

not adequate to rely on the angle of those moments to robustly compute shape orientation, since when the magnitude of those moments is small, their phase is very sensitive to noise.

Moment-based orientation was later addressed by using Generalized Complex (GC) moments [8]. The GC moment of order $(p, q)$ of a polar-coordinate expressed image $f(r, \theta)$ is of the form $G C_{p q}=\int_{0}^{2 \pi} \int_{0}^{\infty} r^{p} e^{j q \theta} f(r, \theta) r d r d \theta$, where $p \in\{0,1,2, \ldots\}$ and $q \in\{1,2,3, \ldots\}$. GC moments, simply termed rotational moments in a previous review (including Legendre, Zernike, and CMs) [11], can be seen as the coefficients of the Fourier series of radial projections of the image. For a shape $\mathbf{z}$, the GC moments collapse into more general sums than the ones in (8):

$$
G C_{p q}(\mathbf{z})=\sum_{n=1}^{M}\left|z_{n}\right|^{p} e^{j q \arg z_{n}} .
$$

To deal with ambiguities that arise when attempting to define and compute shape orientation from a single moment of a particular order, reference [8] uses three non-zero GC moments, with a fixed index $p$. The method is not simple: from $G C_{p q_{1}}$ and $G C_{p q_{2}}$, it is inferred the possibility that the shape is rotationally symmetric; in case there is that possibility, the unambiguous detection of symmetry requires an exhaustive search; if the shape is classified as rotationally symmetric, a third moment $G C_{p q_{3}}$ is also used to compute the orientation. A simple example shows that this method may fail: consider $\mathbf{z}_{1}=[1,-1 / 4,-3 / 4]^{T}$ and $\mathbf{z}_{2}$ its reflection, i.e., $\mathbf{z}_{1}$ rotated by $\pi$, $\mathbf{z}_{2}=-\mathbf{z}_{1}$, and the choice of GC index $p=1$. Using (9), we get $G C_{1 q}\left(\mathbf{z}_{1}\right)=1+\frac{1}{4} e^{j q \pi}+\frac{3}{4} e^{j q \pi}=1+(-1)^{q}=G C_{1 q}\left(\mathbf{z}_{2}\right)$, showing that it is impossible to distinguish between the orientations of $\mathbf{z}_{1}$ and $\mathbf{z}_{2}$ from the moments $G C_{1 q}$ (note that the shape in $\mathbf{z}_{1}$ and $\mathbf{z}_{2}$ is not rotationally symmetric, thus different orientation angles $\theta\left(\mathbf{z}_{1}\right)$ and $\theta\left(\mathbf{z}_{1}\right)$ must be computed). Although reference [8] proposes to tune the index $p$ by maximizing a so-called alternating energy of the radial projection (which also requires exhaustive search), this method fails to exclude $p=1$ for the example above.

\section{PRINCIPAL MOMENTS ANALYSIS (PMA)}

We define and compute $\theta(\mathbf{z})$ using what we call the Principal Moments (PMs) [14] of $\mathbf{z}$. The $k^{\text {th }}$-order PM is defined by

$$
M_{k}(\mathbf{z})=z_{1}^{k}+z_{2}^{k}+\cdots+z_{N}^{k}=\sum_{n=1}^{N} z_{n}^{k},
$$

where $k \in\{1,2,3, \ldots\}$. PMs are easily related to CMs and GC moments, through $M_{k}(\mathbf{z})=C_{k 0}(\mathbf{z})=G C_{k k}(\mathbf{z})$ and the first $N$ of them suffice to describe univocally the underlying shape [15].

The choice $\theta(\mathbf{z})=\arg M_{1}(\mathbf{z})$ (equivalent to the simplest form of the method in [10], using $C_{10}=M_{1}$ ) satisfies (4). In fact, $M_{1}\left(\mathbf{z} e^{j \phi}\right)=M_{1}(\mathbf{z}) e^{j \phi}$, thus $\arg M_{1}\left(\mathbf{z} e^{j \phi}\right)=\arg M_{1}(\mathbf{z})+\phi$. Nevertheless, since $M_{1}(\mathbf{z})=\sum_{n} z_{n}$ is proportional to the shape 
center, its angle is not a characteristic of the shape format, but only of its localization. In practice, to obtain translation invariance, it is common to center the shapes by subtracting the mean $\sum_{n} z_{n} / N$, thus $M_{1}$ becomes zero and useless to determine shape orientation.

The choice $\theta(\mathbf{z})=\arg M_{1}(\mathbf{z})$ is equivalent to imposing the argument of the first-order PM of the rotationally normalized shape (2) to be zero, i.e., $\arg M_{1}\left(\mathbf{z} e^{-j \theta(\mathbf{z})}\right)=0$. Our approach is to generalize this method by doing the same to the $k^{\text {th }}$-order PM:

$$
\arg M_{k}\left(\mathbf{z} e^{-j \theta(\mathbf{z})}\right)=0 .
$$

To solve for $\theta(\mathbf{z})$, use (10) to rewrite (11), $\arg \sum_{n} z_{n}^{k} e^{-j k \theta(\mathbf{z})}=0$. Since complex arguments are defined modulo (mod) $2 \pi$, we then get

$$
\arg \sum_{n=1}^{N} z_{n}^{k}-k \theta(\mathbf{z})+2 \pi l=0,
$$

where $l$ is an integer. The solution for the normalization angle is then

$$
\theta(\mathbf{z})=\frac{\arg M_{k}(\mathbf{z})}{k}+\frac{2 \pi}{k} l, \quad l \in\{0,1, \ldots, k-1\},
$$

where we used (10) again and noted that only $k$ values of $l$ lead to distinct solutions for $\theta(\mathbf{z})$. Expression (13) makes an ambiguity clear, which arises when attempting to define the normalization angle using the $k^{\text {th }}$-order PM alone (for $k \neq 1$ ): there are $k$ distinct values $(\bmod 2 \pi)$ of $\theta(\mathbf{z})$ that annihilate the argument of $M_{k}\left(\mathbf{z} e^{-j \theta(\mathbf{z})}\right)$.

Nevertheless, the normalization angle $\theta(\mathbf{z})$ needs to satisfy (4). We derive what this condition imposes to the solution for $\theta(\mathbf{z})$ that must be picked from the set in (13). By proceeding as in (11)-(12), we express the argument of the $k^{\text {th }}$-order PM of a rotated shape as

$$
\arg M_{k}\left(\mathbf{z} e^{j \phi}\right)=\arg M_{k}(\mathbf{z})+k \phi+2 \pi \hat{l},
$$

where $\hat{l}$ is an integer that guarantees that the arguments of $M_{k}\left(\mathbf{z} e^{j \phi}\right)$ and $M_{k}(\mathbf{z})$ fall within the desired interval, e.g., $(-\pi, \pi]$. Using (14) and (13), we obtain the normalization angle of the rotated shape:

$$
\theta\left(\mathbf{z} e^{j \phi}\right)=\frac{\arg M_{k}(\mathbf{z})}{k}+\phi+\frac{2 \pi}{k}(l+\hat{l}) .
$$

The verification of (4) hinges on the choice of $l$ in (13). If one simply chooses the same $l$ for all shapes, (15) becomes $\theta\left(\mathbf{z} e^{j \phi}\right)=\theta(\mathbf{z})+$ $\phi+(2 \pi / k) \hat{l}$, showing that (4) is satisfied if and only if $\hat{l}=0(\bmod k)$. However, this can not be guaranteed, since in general (14) requires distinct values of $\hat{l}$ for distinct $\phi$ : just imagine $\phi$ ranging from 0 to $2 \pi$ and note that $\arg M_{k}\left(\mathbf{z} e^{j \phi}\right)$ would exhibit jumps (in order to maintain its value within $(-\pi, \pi])$, at values of $\phi$ spaced by intervals of length $2 \pi / k$ (corresponding to changing the value of $\hat{l}$ ).

The crux of our approach is to define the normalization angle in (13) by selecting a value for $l$ that depends on the shape $\mathbf{z}, l(\mathbf{z})$. To achieve this, we use a supplementary PM, $M_{m}$, with $k$ and $m$ coprime, i.e., $\operatorname{gcd}(k, m)=1$. Our choice for $l(\mathbf{z})$ is based on the arguments of $M_{k}(\mathbf{z})$ and $M_{m}(\mathbf{z})$. For simplicity, denote the argument of the $m^{\text {th }}$-order PM of the normalized shape by $A(\mathbf{z}, l)$, where $l$ stands for the particular integer used in (13). Then,

$$
\begin{gathered}
A(\mathbf{z}, l) \stackrel{\text { def }}{=} \arg M_{m}\left(\mathbf{z} e^{-j \theta(\mathbf{z})}\right)=\arg \sum_{n=1}^{N} z_{n}^{m}-m \theta(\mathbf{z}) \\
=\arg M_{m}(\mathbf{z})-\frac{m}{k} \arg M_{k}(\mathbf{z})-\frac{m}{k} 2 \pi l,
\end{gathered}
$$

where we just used (10) and (13). Since $k$ and $m$ are coprime, it is easy to verify that the set $\{-(m / k) 2 \pi l: l \in\{0,1, \ldots, k-1\}\}$ is the same as $\{(2 \pi / k) l: l \in\{0,1, \ldots, k-1\}\}(\bmod 2 \pi)$. Thus, the set $\{A(\mathbf{z}, l): l \in\{0,1, \ldots, k-1\}\}$ contains $k$ different elements $(\bmod 2 \pi)$ spaced by intervals of length $2 \pi / k$. We propose to unambiguously choose $l(\mathbf{z})$ such that $A(\mathbf{z}, l(\mathbf{z}))$ falls within an arbitrary but fixed interval $I$ of length $2 \pi / k: I=\bigcup_{n=-\infty}^{+\infty} I_{n}$, with $I_{n}=\left\{\lambda: \lambda_{0}+2 \pi n<\lambda \leq \lambda_{0}+2 \pi n+2 \pi / k\right\}, \lambda_{0} \in \mathbb{R}$,

$$
A(\mathbf{z}, l(\mathbf{z})) \in I
$$

The ambiguity in the definition of $\theta(\mathbf{z})$ in (13) is now solved, through the choice of $l(\mathbf{z})$ in (17), but we still have to check that this solution satisfies (4). As derived above, $\theta\left(\mathbf{z} e^{j \phi}\right)$ is given by (15), now with $l$ dependent on the shape, i.e., with $l\left(\mathbf{z} e^{j \phi}\right)$, the solution of (17) for the rotated shape $\mathbf{z} e^{j \phi}$. To express the right side of (15) in terms of $\theta(\mathbf{z})$, we must relate $l\left(\mathbf{z} e^{j \phi}\right)$ with $l(\mathbf{z})$. This is done by expressing the argument of the $m^{\text {th }}$-order PM of the normalized rotated shape in terms of the one of the original shape:

$$
\begin{aligned}
& A\left(\mathbf{z} e^{j \phi}, l\left(\mathbf{z} e^{j \phi}\right)\right)= \\
& =\arg M_{m}\left(\mathbf{z} e^{j \phi}\right)-\frac{m}{k} 2 \pi l\left(\mathbf{z} e^{j \phi}\right)-\frac{m}{k} \arg M_{k}\left(\mathbf{z} e^{j \phi}\right) \\
& =\arg M_{m}(\mathbf{z})+m \phi-\frac{m}{k} 2 \pi l\left(\mathbf{z} e^{j \phi}\right)-\frac{m}{k}\left(\arg M_{k}(\mathbf{z})+k \phi+2 \pi \hat{l}\right) \\
& =\arg M_{m}(\mathbf{z})-\frac{m}{k} \arg M_{k}(\mathbf{z})-\frac{m}{k} 2 \pi\left(l\left(\mathbf{z} e^{j \phi}\right)+\hat{l}\right) \\
& =A\left(\mathbf{z}, l\left(\mathbf{z} e^{j \phi}\right)+\hat{l}\right)
\end{aligned}
$$

where (19) uses (16), (20) uses (14), (21) are simple manipulations, and (22) uses (16) again. From (22), we see that $l\left(\mathbf{z} e^{j \phi}\right)+\hat{l}=l(\mathbf{z})$, since only this way $A\left(\mathbf{z} e^{j \phi}, l\left(\mathbf{z} e^{j \phi}\right)\right)=A(\mathbf{z}, l(\mathbf{z})) \in I$ (remember that $I$ does not depend on the shape). Replacing this equality into (15) and using the definition (13), (4) is immediately obtained.

We call our method Principal Moments Analysis (PMA).

The reader may wonder if there are shapes for which all but one PM are zero. However, its easily shown that those shapes do not exist ${ }^{1}$. What may happen is the case when there are not coprime $k$ and $m$ such that $M_{k} \neq 0$ and $M_{m} \neq 0$, i.e., letting $\gamma=\operatorname{gcd}(K), K=\left\{k \in \mathcal{Z}^{+}: M_{k} \neq 0\right\}$, the case when $\gamma>1$. Using results from [14], where PMs are seen as coefficients of a Fourier series, it is simple to conclude that this situation is equivalent to the existence of a $\gamma$-fold rotational symmetry, i.e., that the shape is invariant to rotations of $2 \pi / \gamma$. In this case, all normalization angles of the form $\theta+\hat{k} 2 \pi / \gamma, \hat{k} \in \mathcal{Z}$ lead to the same result. Hence, it suffices to compute an angle $\theta_{\gamma}$ by using PMA with $\theta_{\gamma} / \gamma$ instead of $\theta$ in the derivations of this section and then inverting the scaling effect through the contraction of $\theta_{\gamma}: \theta=\theta_{\gamma} / \gamma$. In terms of the vector $\mathbf{z}$, this procedure is simply equivalent to using the PMs of orders $\gamma k$ and $\gamma m$ instead of the original ones of orders $k$ and $m$.

PMA can be used with any pair of coprime indexes $(k, m)$, provided that $M_{k} \neq 0$ and $M_{m} \neq 0$. To improve robustness, we integrate the contributions of several pairs $\left\{\left(k_{i}, m_{i}\right)\right\}$ by computing pairwise estimates $\theta_{i}(\mathbf{z})$, and defining a robust normalization angle $\theta(\mathbf{z})$ as the (angular) weighted average of them, $\theta(\mathbf{z})=\arg \sum_{i} p_{i} e^{j \theta_{i}(\mathbf{z})}$. The reason for the angular average is its ability to deal with angles close to the region of circular discontinuity (we want the average of $0.1^{\circ}$ and $359.9^{\circ}$ to be $\arg \left(\exp \left(j 0.1^{\circ}\right)+\exp \left(j 359.9^{\circ}\right)\right)=0^{\circ}$, instead of $\left.\left(0.1^{\circ}+359.9^{\circ}\right) / 2=180^{\circ}\right)$. The proof that property (4) holds for the averaged normalization angle is trivial and omitted.

${ }^{1}$ Hint: Consider that all but one of first $N$ PMs are zero. Compute the shape these PMs specify and, from the shape, compute the remaining (higher order) PMs. It will be clear from the result that they cannot be all zero. 


\section{EXPERIMENTS}

To illustrate robustness to noise, we focus on the most challenging scenario of rotational symmetry. We use noisy versions of a threefold rotationally symmetric shape, see the top row of Fig. 2, and normalized their orientations using PMA, obtaining the visually correct results on the bottom. To illustrate robustness to shape sampling density, we used Japanese characters. We synthesized corrupted versions of those characters by removing up to $95 \%$ of the shape points, obtaining shape vectors $\mathbf{z}$ in (1) of very distinct cardinality that were then processed using PMA. In Fig. 3, we single out instances of a specific character to illustrate the consistent orientations obtained.
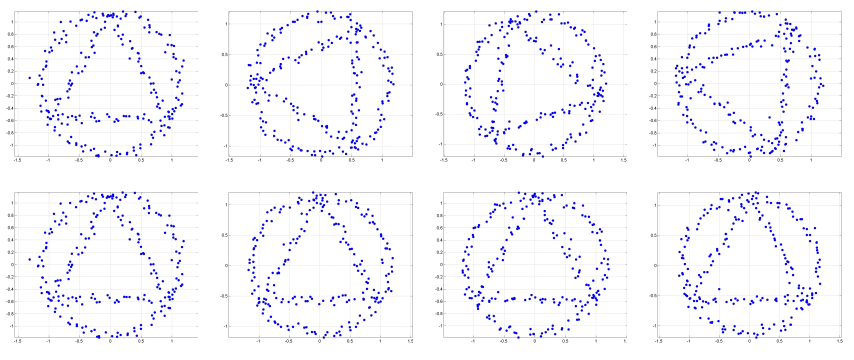

Fig. 2. PMA results. Top: input; bottom: normalized shapes.
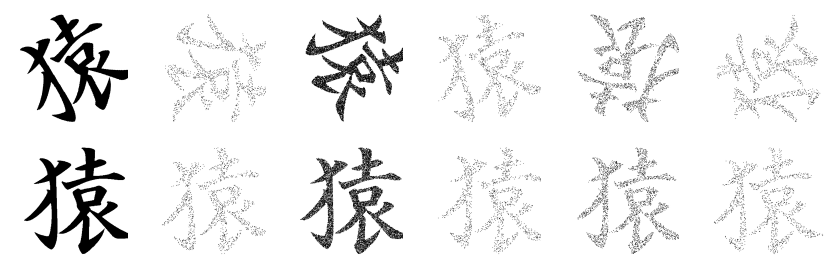

Fig. 3. Using PMA with corrupted Japanese characters.

We also tested PMA with greylevel images, using photos of trademark logos, see examples in Fig. 4 (PMA copes with continuous images through the usual definition of complex moments for this case). In spite of geometric distortions (e.g., perspective, radial) and intensity disturbances, we got consistent results, as illustrated by the examples in Fig. 4. Since we detailed in Sec. 3 several situations where current methods fail, we do not report here experiments with those algorithms. In fact, it would be easy to produce examples where PMA outperforms other methods (just imagine using shapes similar to the ones in Fig. 1). However, we found it would be much more informative to present the discussion in Sec. 3 regarding the core limitations of those methods, i.e., to show how they attempt to use information that is not available in general, than to blindly report sample experiments to support our approach.

\section{CONCLUSION}

We presented a method to compute a unique orientation for arbitrary $2 \mathrm{D}$ shapes. This enables performing rotational normalization. Our method is based on the analysis of particular complex moments of the shape, thus we call it PMA - Principal Moments Analysis. Besides theoretically sound, PMA results robust to disturbances.

\section{REFERENCES}

[1] P. Besl and N. McKay, "A method for registration of 3-D shapes," IEEE T-PAMI, vol. 14 , no. 2,1992
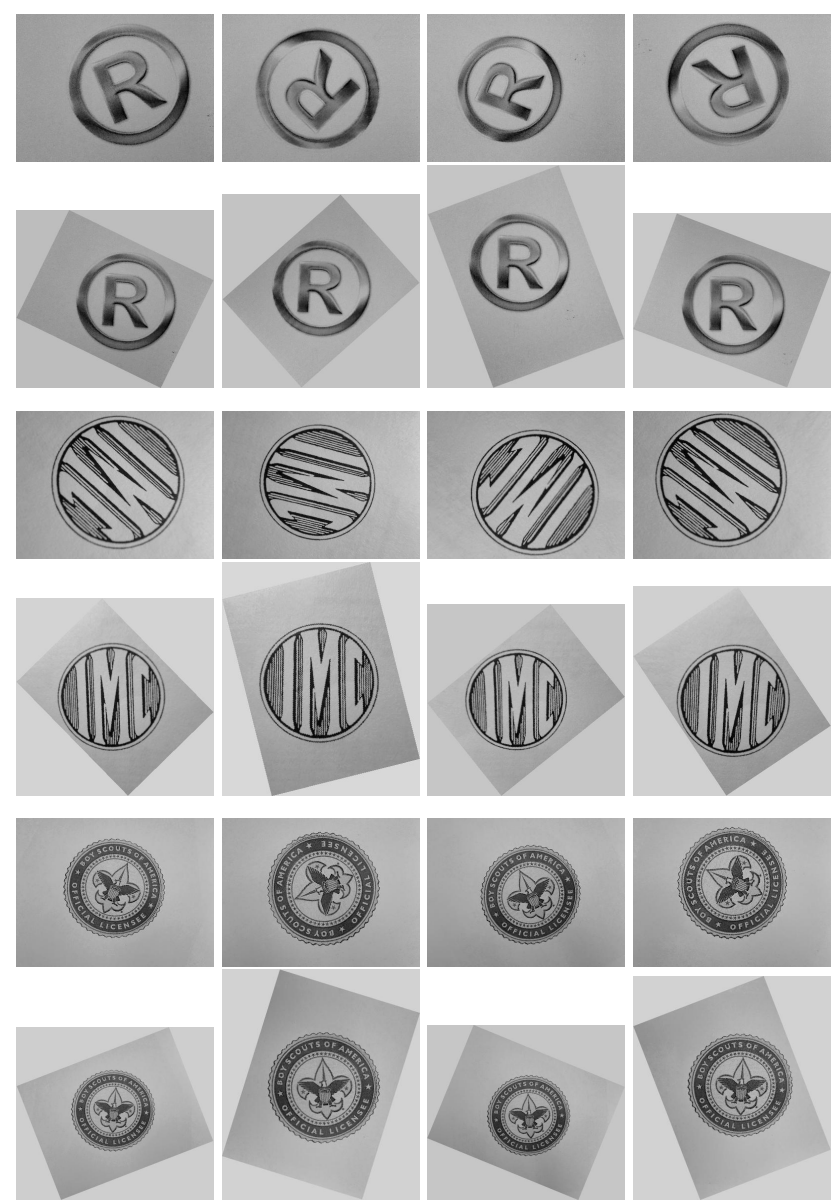

Fig. 4. Using PMA with gray-scale photos of trademark logos.

[2] G. McNeill and S. Vijayakumar, "Hierarchical procrustes matching for shape retrieval," in IEEE CVPR, 2006.

[3] T. Jebara, "Images as bags of pixels," in IEEE ICCV, 2003.

[4] J. Rodrigues, P. Aguiar, and J. Xavier, "ANSIG - an analytical signature for permutation-invariant two-dimensional shape representation," in IEEE CVPR, 2008.

[5] G. Marola, "On the detection of axes of symmetry of symmetric and almost symmetric planar images," IEEE T-PAMI, vol. 11, no. 6, 1989.

[6] J-C. Lin, "Universal principal axes: an easy-to-construct tool useful in definig shape orientations for almost every kind of shape," Pattern Recognition, vol. 26, no. $4,1993$.

[7] J. Zunic, L. Kopanja, and J. Fieldsend, "Notes on shape orientation where the standard method does not work," Pattern Recognition, vol. 39, no. 5, 2006.

[8] D. Shen and H. Ip, "Symmetry detection by Generalized Complex (GC) moments: a closef-form solution," IEEE T-PAMI, vol. 21, no. 5, 1999.

[9] V. Prasad and B. Yegnanarayana, "Finding axes of symmetry from potential fields," IEEE T-IP, vol. 13, no. 12, 2004.

[10] Y. Abu-Mostafa and D. Psaltis, "Image normalization by complex moments," IEEE T-PAMI, vol. 7, no. 1, 1985 .

[11] C-H. Teh and R. Chin, "On image analysis by the method of moments," IEEE T-PAMI, vol. 10 , no. 4, 1988.

[12] V. Ha and J. Moura, "Efficient 2D shape orientation," in IEEE ICIP, 2003.

[13] R. Kondor, "A novel set of rotationally and translationally invariant features for images based on the non-commutative bispectrum," 2008, arXiv:cs/0701127v3 [CS.CV].

[14] J. Crespo, G. Lopes, and P. Aguiar, "Principal Moments for efficient representation of 2D shape," in IEEE ICIP, 2009.

[15] K. Kanatani, Group Theoretical Methods in Image Understanding, Springer, 1990. 